and the technique of administration. The author is enthusiastically in favour of the tungsten arc, and finds it applicable to most conditions. The remainder of the volume deals with the many groups of diseases in which ultra-violet light treatment is advocated. If we were to advance any general criticism, it is that the value of these records would have been considerably enhanced if we had heard a little more about the failures of the method, for these are as instructive as the successes. The mention of ophthalmological conditions is merely cursory. Cases are related of benefit to iridocyclitis, corneal opacities, and cataract. As far as the first of these is concerned we entirely agree with the author in his opinion that the value of ultra-violet rays may be extremely marked; as far as the last is concerned, we are surprised. The technique recommended is the radiation of the eye "through the closed lids"; and since we agree with the statement found on another page of the book that the shorter rays are completely absorbed by one-tenth of a millimetre of skin, while the longest only penetrate as far as the deeper cells of the epidermis, when we think of the interposed layer of muscle, the tarsal plate, the conjunctiva, and the cornea, we are left wondering how this treatment of cataract can act.

\title{
CORRESPONDENCE
}

\section{HEREDITARY GLIOMA OF THE RETINA}

To the Editor of The British Journal of Ophthalmologi

Sir,-In a previous paper on this subject in the British Journal of Ophthalmologi, January, 1919, page 21, reference was made to the recording of further cases. I have now another case to report.

The patient was a girl, nineteen months of age, whose father now aged 34 years, had his right eye enucleated by Argyll Robertson thirty years ago on account of "something to do with the nerve."

There is no conclusive proof that the father's eye was gliomatous but $I$ think this may be regarded as certain. The case was seen in private and complete details were not obtained.

Yours faithfully,

H. M. Traquair.

Edinburgh,

February 13th, 1928. 